\title{
Thermal Polymerization of Acrylamide by Differential Scanning Calorimetry
}

\author{
K. KISHORE and K. N. SANTHANALAKSHMI, Department of Inorganic \\ and Physical Chemistry, Indian Institute of Science, Bangalore 560012, \\ India
}

\section{Synopsis}

Thermal polymerization of acrylamide was studied by differential scanning calorimetry. Latent heat of fusion $\Delta H_{f}$ and enthalpy of polymerization $\Delta H_{p}$ values were found to be 36 and $-18.0 \mathrm{kcal}$ $\mathrm{mol}^{-1}$, respectively. The overall activation energy $E$ for the polymerization was calculated to be $19 \mathrm{k} \mathrm{cal} \mathrm{mol}^{-1}$ up to $60 \%$ conversion. The added free-radical inhibitor (benzoquinone) was found to desensitize the thermal polymerization of acrylamide suggesting the polymerization to be a free-radical type. The existing rate equation for the-heterogeneous bulk polymerization in the presence of initiators has been modified for the thermally initiated bulk polymerization of acrylamide. The experimental overall $E$ value was found to agree well with the calculated $E$ value when considering only the propagation and termination steps, thereby suggesting the process to be similar to postpolymerization of acrylamide.

\section{INTRODUCTION}

Polymerization of solid vinyl monomers can be initiated by thermal or irradiation techniques. The solid-state polymerization of the vinyl monomers initiated by $\gamma$ and UV radiations have been extensively studied by Chachaty and Forchioni. ${ }^{1-4}$ A survey of the literature showed that the kinetics as well as the energetics of the thermal polymerization of acrylamide have not been studied so far.

In the present studies acrylamide was chosen for the investigation of thermally initiated polymerization using differential scanning calorimetry (DSC). Derived kinetic parameters have been used to understand the mechanism of the polymerization process.

\section{EXPERIMENTAL}

\section{Material}

Acrylamide obtained from West Germany (EGA Chemie; ca. 96\%) and from Poole, England (BDH, ca. 99\%) were used for DSC studies. The EGA Chemie sample was used as such for DSC studies using Perkin-Elmer DSC. The BDH sample was twice recrystallized to further purify the sample. This sample was used to confirm the data obtained with the Perkin-Elmer instrument by using another make of DSC (DuPont, 990 model). This sample was also used to study the effect of benzoquinone on the polymerization process. 


\section{DSC Studies}

DSC thermograms were obtained on the Perkin-Elmer instrument (model DSC 2B). The essential operations of the instrument are described elsewhere. ${ }^{5,6}$ The sample was weighed on a Mettler electrobalance and the area under the thermograms were measured using a planimeter. Thermograms were obtained at heating rates of $1.25,2.5,5.0,10,20$, and $40^{\circ} \mathrm{C} \mathrm{min}^{-1}$ and the range settings used were $1,2,5,10$, and $20 \mathrm{mcal} \mathrm{s}^{-1}$ for full-scale deflection, depending on the heating rate and the weight of the sample used. Samples weighing from 0.5 to $1.2 \mathrm{mg}$ were used and the exact weight of the sample in each run was precisely known to one-thousandth of a milligram. Indium was used as the standard for calibrating the temperature axis and the enthalpy output. Only sealed sample holders were used in all the cases. The fraction polymerized $\alpha$ vs time $t$ and $\alpha$ vs temperature $T$ curves were generated from the thermograms by segmental area measurements. The ratio of the segmental area to the total area at a particular temperature or time gave the $\alpha$ value at that instant. The $\alpha-t$ and $\alpha$ $-T$ curves thus generated, are shown in Figs. 1 and 2, respectively.

The latent heat of fusion $\Delta H_{f}$ and the total enthalpy of the polymerization $\Delta H_{p}$ of acrylamide were measured at the heating rates listed above. The calculations were carried out from the area measurements with respect to indium endotherms for melting. These endotherms were obtained at these same heating rates. Thus 36 sets of data were obtained which gave mean values of $\Delta H_{f}=36$ \pm 0.7 and $\Delta H_{\mathrm{p}}=-17.6 \pm 0.4 \mathrm{kcal} \mathrm{mol}^{-1}$.

The DuPont 990 DSC was operated according to the manufacturer's instructions and polyermization thermograms were obtained at heating rates of 5,10 ,

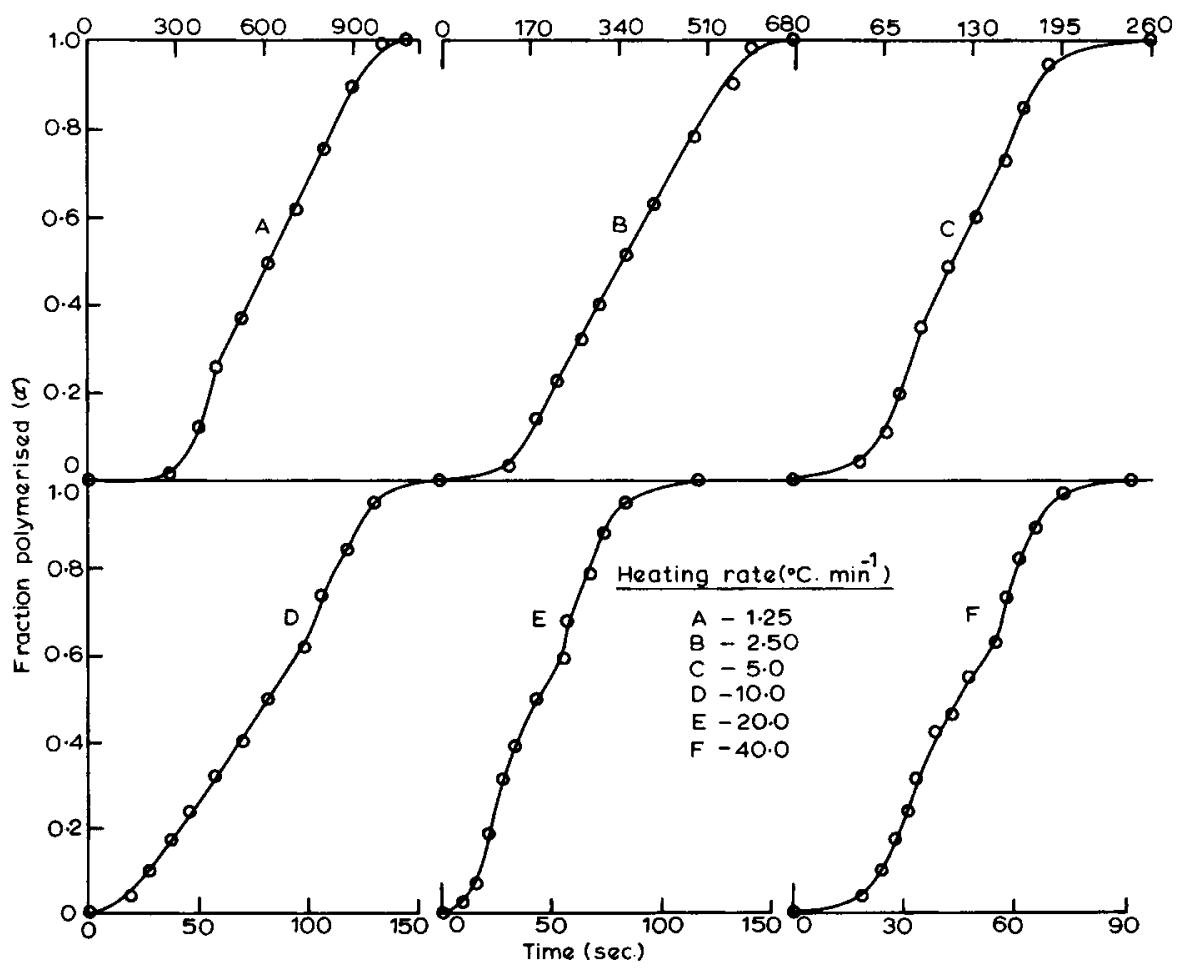

Fig. 1. $\alpha$ vs. time plot for the polymerization of acrylamide. 


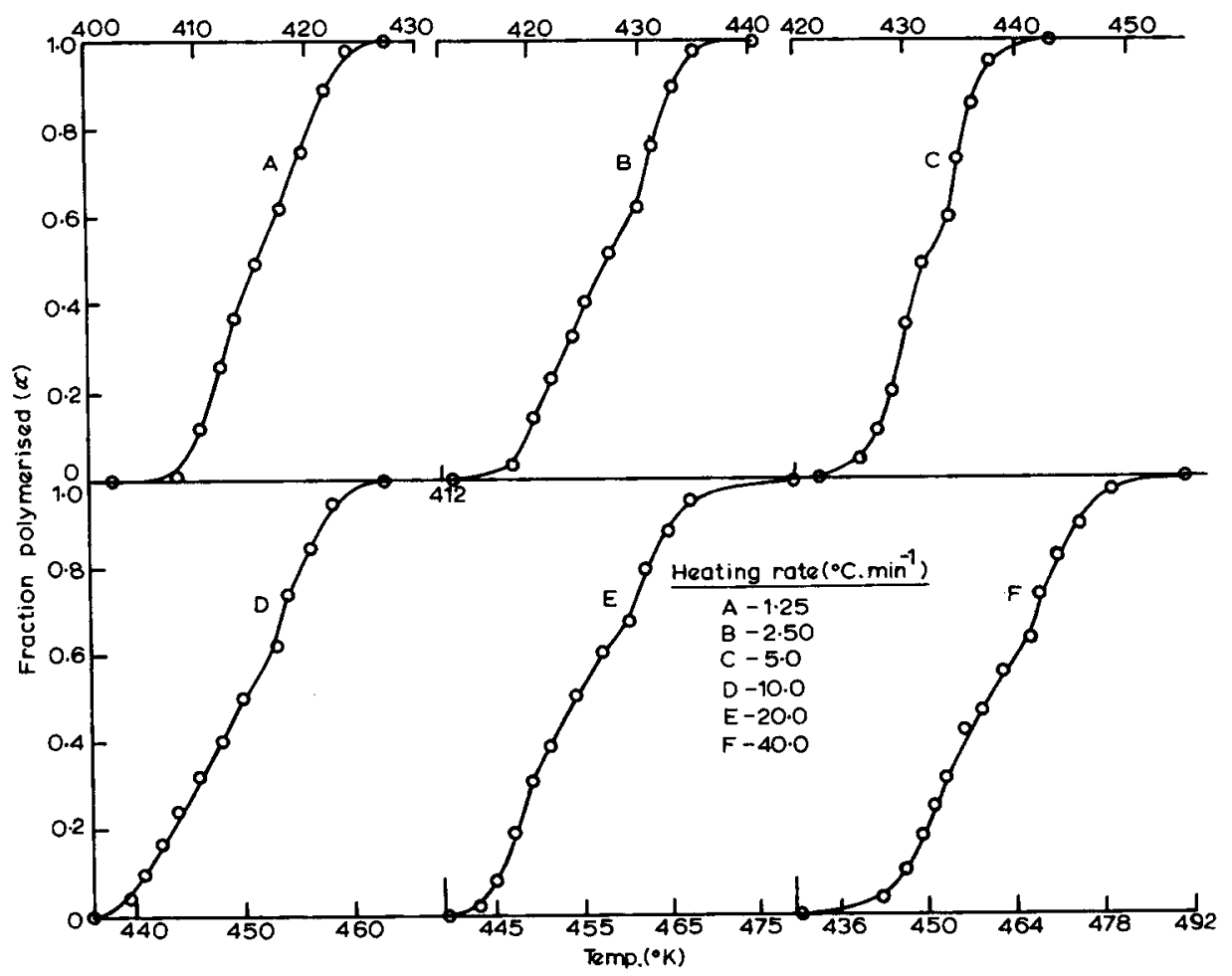

Fig. 2. $\alpha$-Temperature plot for the polymerization of acrylamide.

20 , and $50^{\circ} \mathrm{C} \mathrm{min}^{-1}$ by using the time base operation. The thermograms thus obtained were processed as were the Perkin-Elmer DSC thermograms. The effect of benzoquinone ( $3 \%$ by weight) on the acrylamide polymerization (Fig. 3 ) was tested on the DuPont DSC at a heating rate of $10^{\circ} \mathrm{C} \mathrm{min}-1$.

\section{RESULTS AND DISCUSSION}

When heated, acrylamide first melts and then polymerizes in the molten state. A typical thermogram depicting these processes is shown in Fig. 3. $\Delta H_{f}$ and $\Delta H_{p}$ of acrylamide were estimated to be $36 \pm 0.7$ and $-17.6 \pm 0.4 \mathrm{kcal} \mathrm{mol}^{-1}$, respectively. After melting the sample was further heated; exotherms corresponding to the polymerization process were obtained at different heating rates and are presented in Figs. 4 and 5. The composite nature of the exotherms and a break around $\alpha=0.6$ in both the $\alpha-t$ and $\alpha-T$ plots show the polymerization to be complex in nature.

A method independent of the assumption of the reaction order was used for $E$ calculations. From the $\alpha-T$ plots, the Arrhenius plot was generated using the following equation ${ }^{6}$ :

$$
S / \Delta H_{p}=(1-\alpha)^{n} A e^{-E / R T}
$$

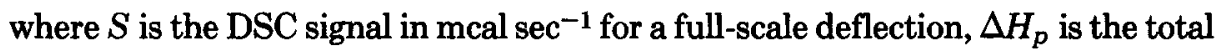
heat of polymerization, $A$ is the frequency factor, and $R$ is the gas constant. Using the eq. (1), $E$ was obtained from the slope of the plot of $\ln S / \Delta H_{p}$ vs. $1 / T$. 


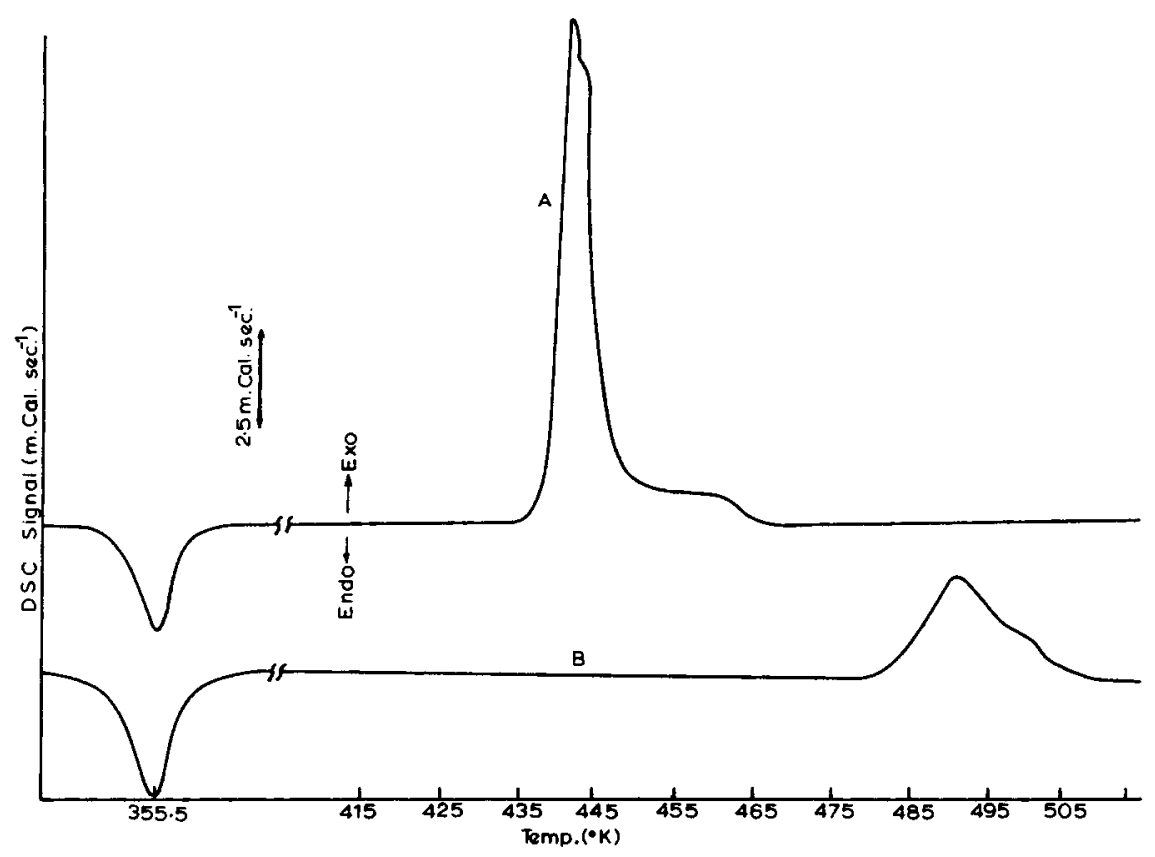

Fig. 3. DSC thermograms of acrylamide in presence and absence of benzoquinone. A: Acrylamide (3.015 mg) $\left(\Delta \mathrm{H}_{\mathrm{p}}=-17.9 \mathrm{kcal} \mathrm{mol}{ }^{-1}\right) \mathrm{B}:$ Acrylamide $(4.950 \mathrm{mg})+$ Benzoquinone $(0.149 \mathrm{mg})\left(\Delta \mathrm{H}_{\mathrm{p}}\right.$ $=-11.5 \mathrm{kcal} \mathrm{mol}^{-1}$ )

In doing so, $\alpha$ and $n$ are kept constant. For a fixed value of $\alpha$, the constancy of $n$ was checked from the so-called reduced-time plot. ${ }^{7}$ The $\alpha$ vs reduced-time plot is shown in Fig. 6. The reduced-time plot shows that the general kinetic pattern of the reaction is independent of the heating rate employed, suggesting thereby that $n$ could be considered constant for all practical purposes. Using eq. (1), $E$ and $A$ are estimated at different $\alpha$ values and are presented in Table I. The $E$ values were also obtained as described above for the recrystallized sample using the Dupont 990 DSC. Good agreement was found to exist in both the cases where the sample and instrument have both been changed.

Considering the first portion of the curve, which gives $E \sim 19 \mathrm{kcal} \mathrm{mol}^{-1}$, the process corresponds to the formation of linear polyacrylamide. This was confirmed from the fact that no ammonia evolution occurs at this part of the reaction. As the polymerization advances the viscosity increases, thereby bringing down the mobility of the molecules. This results in the decrease of $A$ values as shown in Table I. This decrease in $A$ values confirms Flory's prediction of the thermal polymerization. 8 Table I shows that $E$ remains constant up to $\alpha=0.6$ and decreases with further increase in $\alpha$. The exact explanation for this change of $E$ value is not clearly known at present. However, it might be due to the crosslinking of the linear chains.

The present studies on acrylamide polymerization represent a typical thermal bulk polymerization (in absence of initiators) where the polymerization occurs in a heterogeneous medium since the polymer is insoluble in the molten monomer. The rate $R_{p}$ of thermally initiated homogeneous polymerization of vinyl monomers has been given as follows. ${ }^{8}$

$$
R_{p}=k_{p}\left(k_{i} / k_{t} 1 / 2\right)[M]^{2}
$$



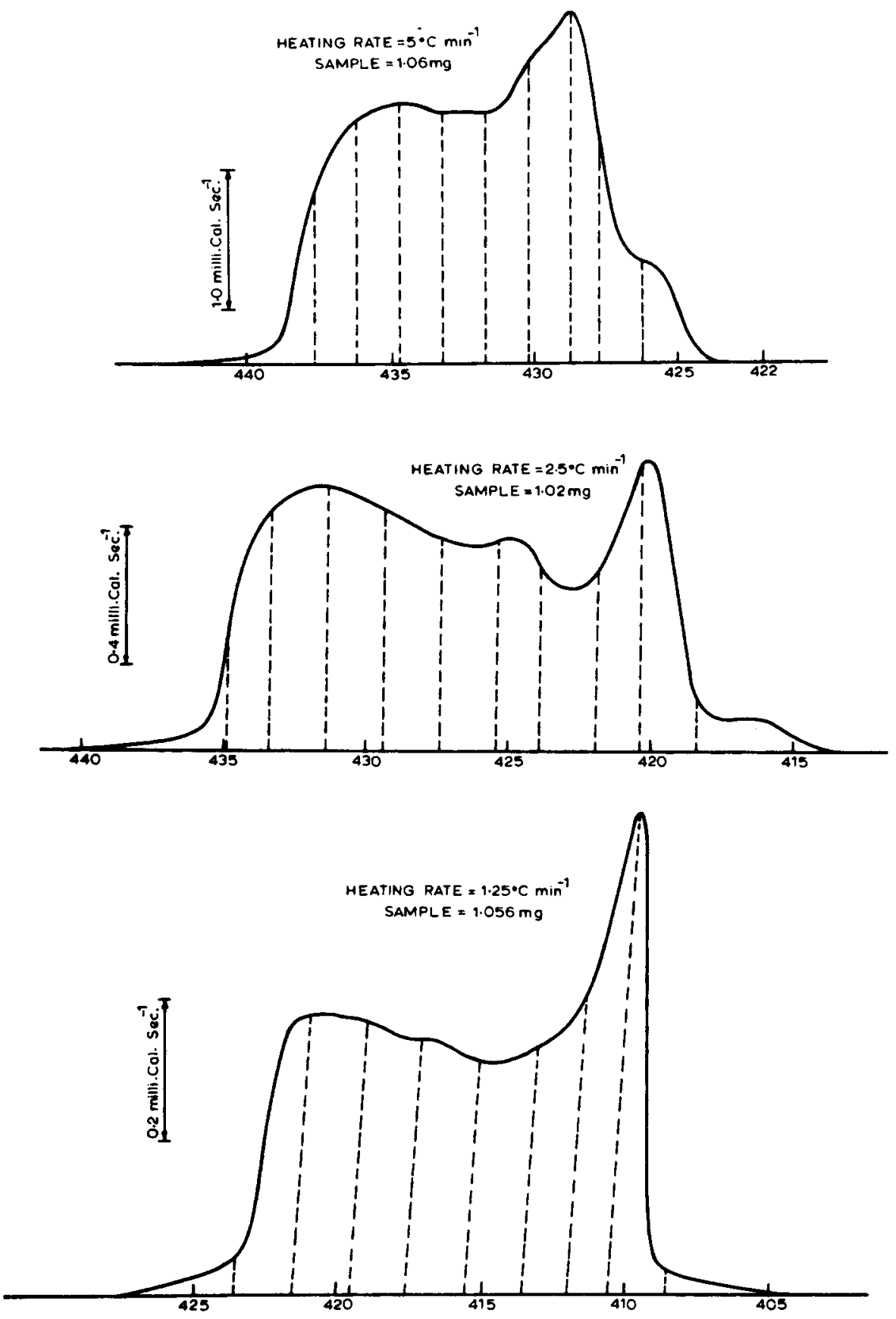

Fig. 4. DSC Thermograms for the polymerization of acrylamide.

where $k_{p}, k_{i}$, and $k_{t}$ are the rate constants of the propagation, initiation, and termination steps and $[M]$ is the monomer concentration. For heterogeneous polymerization of vinyl monomers (in the presence of an added initiator) the following rate equation has been given wherein the polymerization in the homogeneous liquid and in the precipitated polymer phases have been considered simultaneously. ${ }^{9,10}$ 

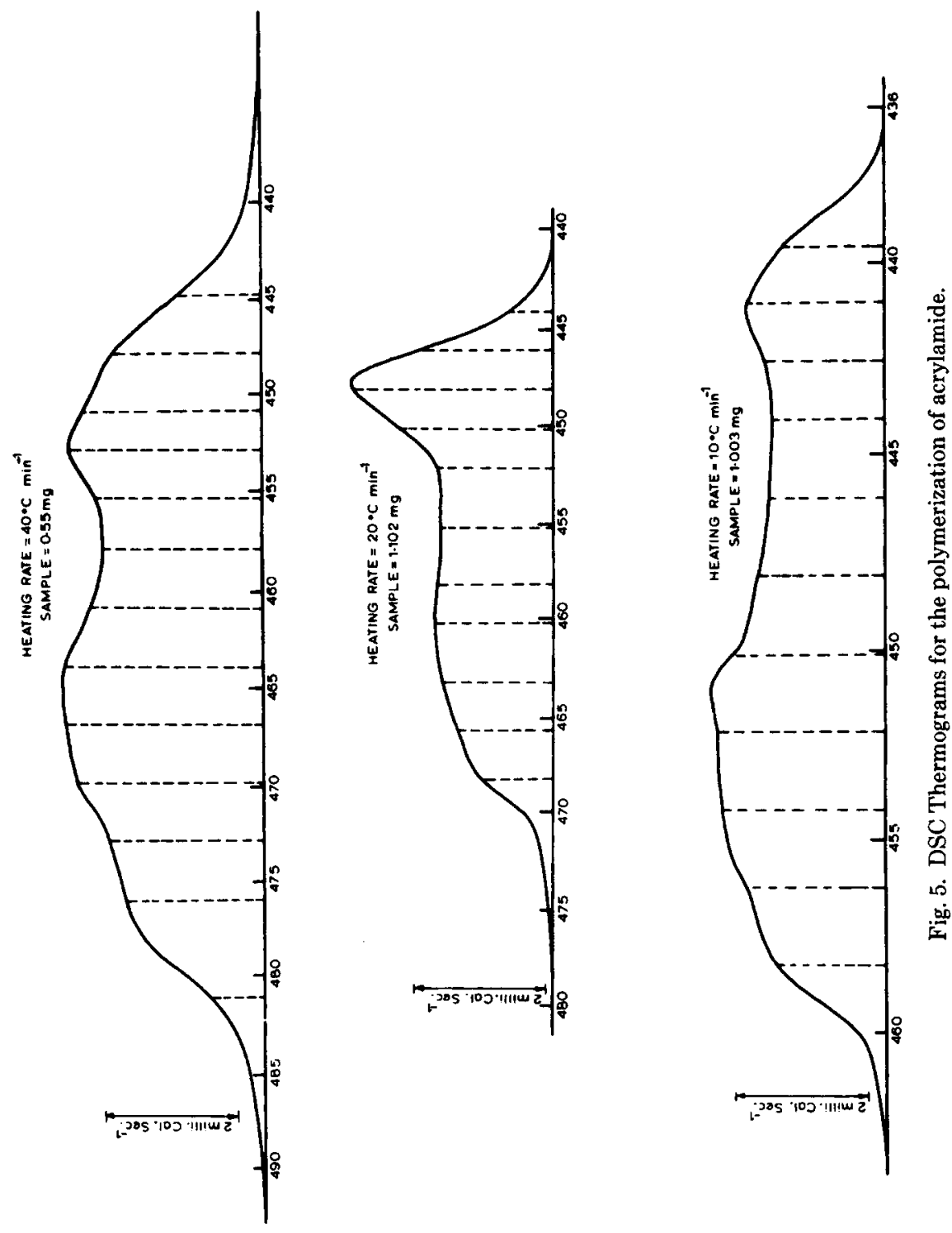


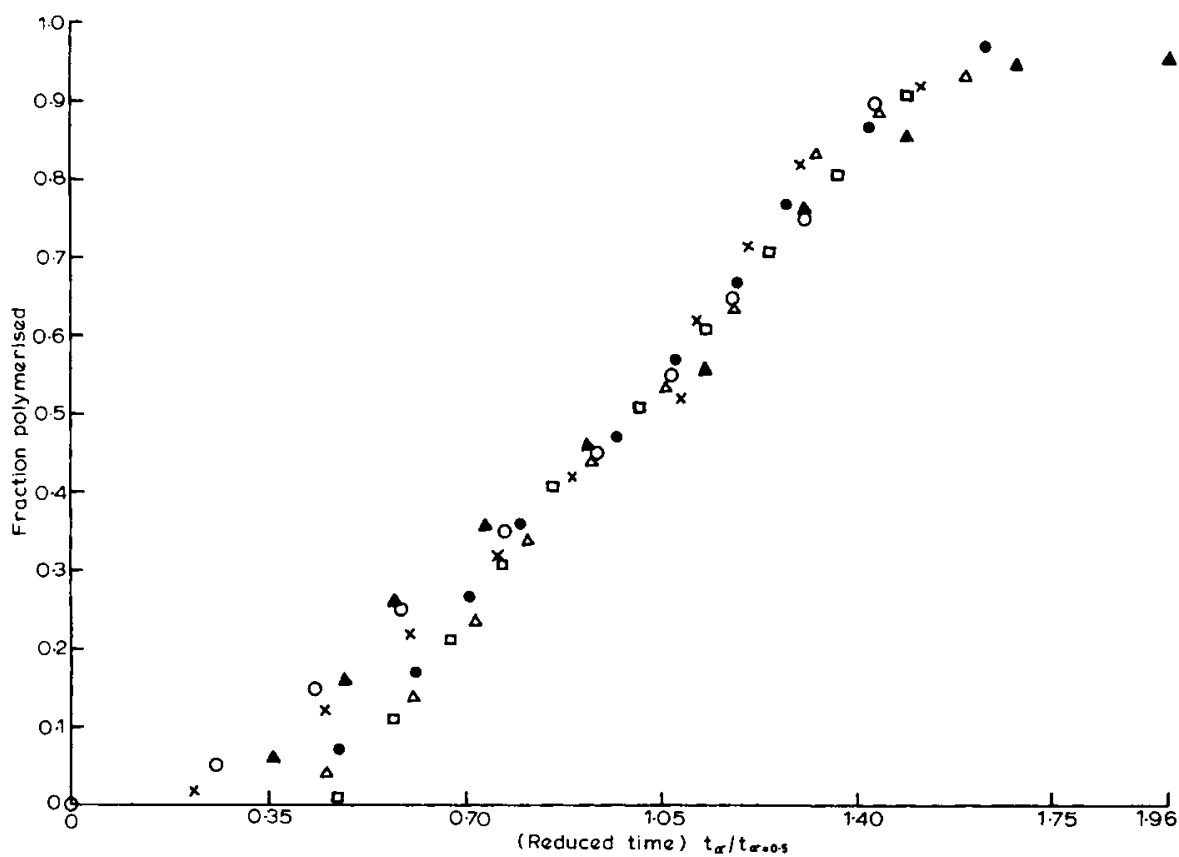

Fig. 6. Fraction polymerized vs. reduced time at various stages of reaction. Heating rate ${ }^{\circ} \mathrm{C} / \mathrm{min}$; (ㅁ) $1.25 ;(\mathrm{x}) 2.50 ;(\Delta) 5.0 ;(0) 10.0 ;(\Delta) 20.0 ;(\bullet) 40.0$.

$$
R_{p}=k_{p}\left(f k_{d}[I] / k_{t}\right)^{1 / 2}\{[M]+f(p)\}
$$

where $f$ is the initiator efficiency, $k_{d}$ is the rate constant for initiator dissociation, and $[I]$ is the initiator concentration and function $f(p)$ is proportional to the polymer concentration $[p]^{x}(x=1$ at low conversions and $x=2 / 3$ at high conversions for the PVC polymerization). In the present case of thermally initiated bulk polymerization of acrylamide wherein the insoluble monomer precipitates out, the rate equation (3) can be used with proper modifications. Making use of eqs. (2) and (3), the rate equation for the thermally initiated (without any initiator) heterogeneous bulk polymerization of acrylamide can be written

$$
R_{p}=k_{p}\left(k_{i} / k_{t}\right)^{1 / 2}\left\{[M]^{2}+f(p)\right\}
$$

Here, the function $f(p)$ is proportional to $[p]^{n}$, where $n$ is an empirical factor similar to the order of the reaction which becomes unity at lower conversions.

TABLE I

Kinetic Parameters for the Polymerization of Acrylamide

\begin{tabular}{ccc}
\hline & $\begin{array}{c}K \\
\left(\mathrm{kcal} \mathrm{mol}^{-1}\right)\end{array}$ & $\begin{array}{c}A \\
\left(\mathrm{~s}^{-1}\right)\end{array}$ \\
\hline 0.1 & 19.5 & $6.8 \times 10^{4}$ \\
0.2 & 19.6 & $6 \times 10^{4}$ \\
0.3 & 20.5 & $1.7 \times 10^{4}$ \\
0.4 & 18.2 & $1.4 \times 10^{4}$ \\
0.5 & 18.5 & $1.1 \times 10^{4}$ \\
0.6 & 17.5 & $0.7 \times 10^{4}$ \\
0.7 & 15.6 & $0.01 \times 10^{4}$ \\
0.8 & 14.8 & $\ldots$ \\
0.9 & 11.9 & $\ldots$ \\
\hline
\end{tabular}


At any instant, $[p]$ is equal to the extent of the reaction $\alpha$ which is given in terms of $[M]$ as follows:

$$
1-[M]=\alpha=[p]
$$

Substituting $f(p)$ in terms of $[M]$, eq. (4) becomes

$$
R_{p}=k_{p}\left(k_{i} / k_{t}\right)^{1 / 2}\left\{[M]^{2}+k(1-[M])^{n}\right\}
$$

where $k$ is a proportionality constant that can be determined experimentally. At low polymer conversion $[m]$ is much larger than $[p]$, so the term $k(1-[M])^{n}$ becomes negligible, resulting in eq. (2). At higher conversions, the term $[M]^{2}$ becomes negligible in comparison with the term $k(1-[m])^{n}$, resulting in a rate equation of the form

$$
R_{p}=k_{p}\left(k_{i} / k_{t}\right)^{1 / 2} k\{1-[M]\}^{n}
$$

It is obvious that both eq. (2) and eq. (7) give the same overall $E$ for the polymerization process,

$$
E=E_{p}+1 / 2 E_{i}-1 / 2 E_{t}
$$

where $E_{p}, E_{i}$, and $E_{t}$ are the activation energies for the initiation, propagation, and termination steps.

All the above rate expressions, eqs. (2)-(8), are exclusively valid only for free-radical polymerization. Hence, in the present case one has to prove that acrylamide polymerization occurs via a free-radical mechanism before making use of the above expressions. To do so, the following simple experiment was carried out. The DSC thermograms in the presence and absence of benzoquinone (Fig. 3) very clearly show that the polymerization is desensitised drastically because of the inhibition of the free radicals produced. Attention may be drawn to the fact that the melting endotherm remains unaffected in the presence of benzoquinone, while only the polymerization exotherm is affected. In Fig. 3, the inhibition of the polymerization is further shown by flattening of the sharp exothermic peaks and reduction in the heats of polymerization. These facts conclusively prove that acrylamide polymerization occurs via a free-radical mechanism.

Chachaty and Forchioni ${ }^{4}$ have reported the $E_{p}, E_{t}$, and $E$ values to be 3538 , and $18 \mathrm{k} \mathrm{cal} \mathrm{mol}^{-1}$, respectively, for the $\gamma$-irradiated solid-state postpolymerization of acrylamide. They have justified their $E$ value by the equation

$$
\Delta T_{\text {crit }}=1.37 R T_{e}{ }^{2} / E
$$

where $\Delta T_{\text {crit }}$ is the critical difference temperature, $T_{e}$ is the external temperature, and $E$ the overall activation energy. One can obtain another justification for the postpolymerization process from eq. (8) by considering only the $E_{p}$ and $E_{t}$ values and excluding the $E_{i}$ value. Thus

$$
E=E_{p}-1 / 2 E_{t}
$$

Substituting the values for $E_{p}$ and $E_{t}$ into (10), the overall $E=16 \mathrm{kcal} \mathrm{mol}^{-1}$ agrees with the reported value of Chachaty and Forchioni. ${ }^{4}$

In the present work $E=19 \mathrm{kcal} \mathrm{mol}^{-1}$ has been obtained for the polymerization of acrylamide to form linear polyacrylamide. The implications of this $E$ value might be discussed as follows. The overall $E$ for the polymerization process 
can be calculated by using eq. (8). The $E_{i}$ can be calculated by considering the dissocation of a $\pi$ bond yielding two $\sigma$ bonds, resulting in monomeric diradicals during the polymerization, and with liberation of $60 \mathrm{kcal} \mathrm{mol}^{-1}$ of energy. If one considers the formation of dimeric diradicals ${ }^{11}$ the value of $E_{i}$ should be about $30 \mathrm{kcal} \mathrm{mol}^{-1}$. Substituting the reported $E_{p}$ and $E_{t}$ values, $E$ was found to be 46 and $31 \mathrm{kcal} \mathrm{mol}^{-1}$ for the $E_{i}$ values of 60 and $30 \mathrm{kcal} \mathrm{mol}^{-1}$, respectively. These $E$ values are quite different from the experimentally observed value, suggesting that thermal polymerization (as in the present case) does not involve three distinct steps. If one considers only the propagation and termination steps, as in the postpolymerization of acrylamide, ${ }^{4}$ one obtains a very good correlation between the experimental and the calculated values. This suggests that the polyermization exotherm involves only propagation and termination steps and that initiation has occurred much before the inception of polymerization. Attempts were made to detect the presence of free radicals in the melt below the starting temperature of polymerization by ESR, but the experiments are inconclusive so far.

One of the authors (K. K.) wishes to thank the University Grants Commission, New Delhi, India and Institute for Cultural Relations, Budapest for arranging the visit to the Research Laboratory For Inorganic Chemistry, Budapest under the Indo-Hungarian Cultural Exchange Programme; to thank Professor T. Szekeley (Director of the Institute) for providing the facilities for carrying out the Perkin-Elmer DSC measurements; and to thank M. Lengyel for her help in obtaining the DSC thermograms.

\section{References}

1. C. Chachaty and A. Forchioni, J. Polym. Sci. Part, A-1, 10, 1905 (1972).

2. C. Chachaty and A. Forchioni, Polym. Lett., 9, 483 (1971).

3. A. Forchioni and C. Chachaty, J. Polym. Sci. Part A-1, 10, 1923 (1972).

4. C. Chachaty, M. Latimier and A. Forchioni, J Polym. Sci. Part A-1, 13, 189 (1975).

5. K. Kishore, V. R. Pai Verneker, and V. K. Mohan, Thermo chim. Acta, 13, 277, (1975).

6. K. Kishore, Anal. Chem., 50, 1079 (1978).

7. K. Kishore, Ind. J. Chem., 16A, 553 (1978).

8. P. J. Flory, Principles of Polymer Chemistry, Cornell University, Ithaca, NY, 1953, pp. $129-131$.

9. F. W. Billmeyer, Jr., Text book of Polymer Science, 2nd ed., Wiley, New York, 1971 pp. $357-8$

10. H. S. Mickley, A. S. Michaels, and A. L. Moore, J. Polym. Sci, 60, 121 (1962).

11. Ref. 8. pp. 130-2. 